\title{
De la inmunología a la infectología en Honduras: Una mirada al desarrollo conjunto de ambas especialidades desde la perspectiva de la medicina clínica
}

From immunology to infectology in Honduras: A look at the joint development of both specialties from the perspective of clinical medicine.

\author{
Jorge Alberto Fernández Vásquez,, ${ }^{1,2}$ Jackeline Alger. ${ }^{2,3}$ \\ ${ }^{1}$ Médico Especialista en Alergia e Inmunología Clínica; Hospital y Clínicas Viera; Centro de Salud Integral Zoé \\ ${ }^{2}$ Instituto de Enfermedades Infecciosas y Parasitología Antonio Vidal; Tegucigalpa. \\ ${ }^{3}$ Médica, Doctorado (PhD) en Parasitología; Unidad de Investigación Científica, Facultad de Ciencias Médicas, UNAH; Departamento de Laboratorio Clínico, \\ Hospital Escuela.
}

RESUMEN. Esta revisión bibliográfica se realizó con el propósito de brindar una perspectiva histórica que relacione la estrecha vinculación de la infectología y la inmunología y sus repercusiones en la medicina en Honduras, haciendo relación a la práctica médica desde sus orígenes difusos de la época colonial hasta la actualidad. Se revisó la información de los registros de la Revista Médica Hondureña y publicaciones similares nacionales. Se presentan y discuten aspectos clínicos, de salud pública, académicos y éticos en el modo de actuar profesional donde convergen ambas especialidades, resaltando elucubraciones, actualizaciones contextuales, procesos, instituciones y figuras médicas que han marcado en forma indeleble su desarrollo y evolución.

Palabras clave. Alergia e inmunología, Honduras, Infectología, Medicina clínica.
Recibido: 03-02-2020; Aceptado para publicación 14-04-2020

Dirección para correspondencia: Dr. Jorge A. Fernández

Correo electrónico: joralferv@gmail.com

Declaración de relaciones y actividades financieras y no financieras y conflictos de interés: ninguna.

DOI: https://doi.org/10.5377/rmh.v88iSupl.1.11676 ...el bacilo de la peste no muere ni desaparece jamás, que puede permanecer durante decenios dormido en los muebles, en la ropa, que espera pacientemente en las alcobas, en las bodegas, en las maletas, los pañuelos y los papeles, $y$ que puede llegar un día en que la peste, para desgracia y enseñanza de los hombres, despierte a sus ratas y las mande a morir en una ciudad dichosa.

Albert Camus, La Peste.

\section{INTRODUCCIÓN}

Aunque los relatos históricos más antiguos nos remiten a grandes epidemias que fulminaron millones de personas, ese comportamiento de relaciones entre especies infecciosas interactuando con el sistema de respuesta y defensa del ser humano, apenas lo que se pudo reconocer en milenios de historia, es que la enfermedad se aplacaba cuando la mayor parte de la población había sufrido los embates del mal, al tiempo que disminuía el número de nuevas víctimas (susceptibles), dejando alta mortalidad y sobrevivientes que quedaban naturalmente inmunizados. Pero no es sino con las experiencias de Jenner, provocando inmunidad contra la viruela, que comienza la observación científica de la defensa corporal contra fuentes externas, identificados posteriormente como gérmenes patógenos o agentes infecciosos; siendo los estudios minuciosos de Pasteur, Koch, Erlich y otros investigadores, los que sentaron las bases para el desarrollo de la Infectología y la Inmunología, alrededor de la segunda mitad del siglo XIX y giro del XX. Louis Pasteur se dio a la batalla de convencer al mundo científico de la validez de su teoría germinal. Su comunicación en 1880, de que la inmunización profiláctica contra el cólera de las aves era posible, marcó el inicio de la Inmunología y la Infectología científicas. ${ }^{1}$

Los acelerados descubrimientos en la identificación de gérmenes y de los productos celulares, tisulares y plasmáticos de la respuesta inmunológica, con la puesta a punto de diversas técnicas de laboratorio, abrieron paso a la aplicación 
clínica en el diagnóstico y tratamiento, así como medidas sanitarias de epidemiología y de medicina preventiva a través de vacunaciones y estudios ambientales y de productos de consumo humano como bebidas y alimentos. Durante el último cuarto del siglo XIX y primero del siglo XX, en Honduras los conocimientos de ambas especialidades emanaban de los centros asistenciales y de formación como el Hospital General de Tegucigalpa y la Facultad de Medicina de la UNAH, que recién fundados, recibían la influencia de la medicina europea y del noreste de los Estados Unidos. Esta evidencia se vio matizada por la que luego vendría de las escuelas latinoamericanas como México, Argentina, Chile y Brasil, acrisolando una amalgama de medicina clínica que se vería beneficiada por los aportes vertiginosos y universales de la Microbiología y la Inmunología. De otro lado, el establecimiento de los enclaves bananeros en la costa norte del país trajo consigo el desarrollo de centros de salud y hospitales de las empresas bananeras, que hicieron importantes aportes a la medicina tropical. ${ }^{2,3}$ La aparición de la Revista Médica Hondureña (RMH) en 1930, marcó un hito extraordinario en la compilación de escritos médicos locales e internacionales que dejaron evidencia del accionar clínico en los diferentes momentos de la evolución de la práctica médica. Existen pocas referencias sobre publicaciones médicas anteriores a la creación de la RMH. Se pueden encontrar algunos artículos en diarios y en la Revista del Archivo y Biblioteca Nacionales de Honduras, las cuales tuvieron una existencia efímera además de la Revista de la Juventud Médica Hondureña, la Revista Médico Quirúrgica (ca. 1920), y Medicina Clínica (1986). Sin embargo, sólo la RMH logró mantenerse viva para recoger la crónica de la producción científica y humanística médica hondureña. ${ }^{4}$

En un afán de describir conocimientos, ideas y prácticas en la medicina hondureña, que hacen converger ambas especialidades, aunque se involucran otras que son afines 0 están íntimamente vinculadas, se ha hecho una revisión de publicaciones científicas nacionales y algunas extranjeras, libros de historia de la medicina en el país, y entrevistado a algunos profesionales muy relacionados a la práctica de estas especialidades. De esta manera, se ubican la mayoría de los datos a partir de los años 30's del siglo XX, cuando surge la Revista Médica Hondureña como plataforma de expresión de conocimientos y experiencias mundiales y nacionales, ${ }^{5}$ reforzándose con nuevas publicaciones a lo largo de la segunda mitad de ese mismo siglo. Este relato se ha hecho con el propósito de brindar una perspectiva histórica que relacione la estrecha vinculación de estas especialidades y sus repercusiones en la medicina nacional. Para ello se ha tratado de recoger la información científica más asequible, particularmente la que figura en la RMH y otras publicaciones similares, haciendo uso de las bases de datos de la Biblioteca Médica Nacional y del Colegio Médico de Honduras, y de algunas colecciones de revistas científicas y buscadores internacionales como Scielo, Google Scholar, y otros.

\section{DE LA INMUNOLOGÍA A LA INFECTOLOGÍA EN HONDURAS}

La escasa información de la medicina precolombina y de la primera época de la colonia española, figura en algunos textos de la historia de la medicina en Honduras que, en términos globales en relación con este relato, se enmarca en la descripción de las grandes epidemias que azotaron la provincia en aquellos tiempos ${ }^{6,7}$ Apenas si tenemos datos inciertos $y$ vagos sobre "las fiebres remitentes reinantes todo el año en esa región, acentuándose en la estación lluviosa". En general, durante los siglos XVII y XVIII se recogen relatos de epidemias de viruela, cólera, paludismo, fiebre amarilla, sobre todo en las ciudades más importantes como Trujillo, Comayagua y Tegucigalpa, que se combatían con el auxilio de los religiosos como los mercedarios, unos pocos médicos extranjeros, el esfuerzo oficial y la movilización comunitaria. En el siglo XIX el medicamento heroico era la decocción de quina para las fiebres intermitentes y el parasitismo intestinal era tratado con multitud de brebajes compuestos de varias plantas regionales. ${ }^{8}$ En mayo de 1806 estalló la epidemia de viruela justo cuando en el año anterior (1805) había arribado la vacuna a Trujillo (4 niños portadores), por lo que se procedió a la vacunación en las zonas donde brotó, especialmente Comayagua. ${ }^{9}$ Para finales de ese siglo los estudiantes de la novel Facultad de Medicina ya participaban en jornadas de vacunación contra la viruela (1890). El cólera hizo 5 brotes epidémicos durante ese mismo siglo, obligando a las autoridades y la comunidad a tomar estrictas medidas profilácticas y de control. ${ }^{10}$ En 1903 hubo un brote de enfermedades venéreas en prostitutas de Tegucigalpa, por lo que el gobernante Bonilla las confinó en la misma Facultad de Medicina, generando la protesta de sus estudiantes, lo que valió para que ordenara su cierre hasta $1910 .{ }^{11}$

Tras los hallazgos de Pasteur (descubrimiento de la inmunización pasiva de las gallinas respecto del cólera, la vacunación preventiva de las ovejas contra el carbunco, y el tratamiento profiláctico de la rabia), la Inmunología se desarrolló en varias fases hasta llegar a la segunda guerra mundial. Entre estas hay que mencionar el descubrimiento de que los gérmenes muertos y los filtrados de cultivos exentos de microbios son capaces de producir inmunidad; la introducción de la vacunación con gérmenes vivos; el descubrimiento de las toxinas, las antitoxinas y la inmunización pasiva; el descubrimiento de las propiedades y composición de los sueros inmunes; el hallazgo de la fagocitosis y la polémica sobre el mecanismo humoral o celular de los procesos inmunitarios; el descubrimiento de la anafilaxia y la alergia; la etapa fugaz dedicada a la tuberculinoterapia; y, finalmente, la ampliación del concepto de antígeno. ${ }^{12}$

Para esos albores, la serología alcanzó enorme vigencia en el manejo diagnóstico y terapéutico de pacientes, así como la autohemoterapia, reflejando los significados de la ciencia en ese momento. El entusiasmo clínico fue tan grande que se llegaron a realizar ensayos que posteriormente se demostraron inútiles, por no corresponder a la relación lógica del origen de la enfermedad 
y su manejo. Muchas comunicaciones describen diferentes abordajes con derivados serológicos y sanguíneos para estudio y tratamiento de pacientes. Por ejemplo, una comunicación de 1931, relata que "las reacciones de fijación del complemento y de floculación constituyen, en general, un recurso biológico preciso para el diagnóstico de las distintas formas clínicas de la tuberculosis". ${ }^{13}$ Otra puntualiza sobre la prueba de AschheimZondek (detección de gonadotropina coriónica en la orina de mujer) y su aplicación a la diagnosis de preñez, epitelioma coriónico y mola hidatidiforme. ${ }^{14}$ En tanto otra relata que... Desde hace 25 años que fue creada la reacción de Wasserman, como una consecuencia de las famosas experiencias de Bordet-Gengou, el método ha sufrido la crítica y numerosas vicisitudes, $y$, sin embargo, hoy día aún es considerado como uno de los métodos de más valor en el serodiagnóstico de la sifilis, por lo cual debe existir en él, un gran fondo de verdad. Las reacciones de precipitación o floculación son de más reciente introducción pues no cuentan más que con unos 6 años de vida y no obstante son ya las preferidas. ${ }^{15}$ Como experiencia local, el Dr. don Manuel Cáceres Vigil presentó en 1934, al seno de la Asociación Médica, algunos datos sobre reacciones cruzadas entre la prueba estándar de sífilis y malaria. ${ }^{16}$

Respecto a uso de vacunas, suero o sangre en la práctica clínica, hubo por aquella época mucha actividad de ensayos en diversas entidades infecciosas y no infecciosas. Así, para tratamiento de la gonorrea, se planteaba que... Este método (vacunoterapia) es para ellos un medio y no un fin. Los éxitos y fracasos dependerán para los mismos, del antígeno utilizado, del organismo infectado y de la posología instituida. ${ }^{17}$ Para sífilis se realizó Transfusión de Plasma de Sangre con Reacciones Serológicas Positivas por Sifilis. ${ }^{18}$ También se planteó que para tratar la apendicitis aguda: El alistamiento de la sueroterapia a los ataques de apendicitis, constituye una brillante conquista de índole práctica que interesa a todos los médicos. ${ }^{19} \mathrm{O}$, las prácticas en difteria: Nuevas experiencias demostrativas de la curación de la difteria en el cobayo por la Haptinogenina [Méndez], y comparación con la obtenida por el suero antidiftérico. La teoría biológica de la inmunidad de Méndez, que atribuye a los Haptinógenos la propiedad de facilitar al organismo la peptización de los gérmenes patógenos, por la producción de haptinas (anticuerpos), cuyo objeto es la esterilización de ellos; criterio que está muy lejos del modo de pensar clásico-que atribuye el efecto curativo del suero a la neutralización de la toxina por su antitoxina. ${ }^{20}$ Para tratar el sarampión, sobre todo en niños pequeños, se esbozaba que... En virtud de las inmunisinas trasmitidas por la madre a través de la placenta, el niño nace poseedor de una inmunidad hereditaria. Es por ese motivo que la sueroterapia, con el fin de obtener la sueroprevención absoluta, está particularmente indicada en ese período de la vida. ${ }^{21}$ Las nuevas ideas sobre la etiología, evolución y tratamiento de la enfermedad reumática expresaban que... si a la vez que el ataque inflamatorio de las articulaciones ha sido mordido el corazón, entonces el reumatismo poliarticular agudo desplaza definitivamente toda su gravedad hacia la lesión cardíaca; lo articular cede a ésta el rango y primacía. En "La alergia en el reumatismo" (Medicina Latina, marzo de 1933), se relata la descripción de Cañizo con su magistral conferencia sobre "Patología cardiovascular del reumatismo" (Medicina Latina, febrero); o las experiencias de Trías de Bes, que emplea con éxito la vacuna polivalente de los gérmenes aislados de las amígdalas (comunicación a la Academia de Medicina de Barcelona, 20 de marzo de 1933,) y Maurice Louis Vialle en su tesis inspirada por Laubry (Presse Medícale, 20 de junio de 1933), propugnando la vacuna estreptocócica polivalente. Adicionalmente, Strond, Glodsmith y Thorp, en su reciente trabajo "Diez años de observación de niños con afección cardiorreumática", aconsejan el empleo de preparados de estreptococos hemolíticos por vía intravenosa (The Journal of the American Medical Association, 13 de agosto de 1933). ${ }^{22,23}$ Un ejemplo de uso de antisuero en forma no específica se describe en el trabajo El antiveneno botrófico en las diátesis hemorrágicas (doctores Eduardo Selva y Ricardo Aguilar), empleando como último recurso el suero de Barba Amarilla (Antiveneno Botrófico) en los casos de hemorragias en pacientes diatésicos o en pacientes con anemias secundarias producidas por el paludismo y la anquilostomiasis. ${ }^{24}$ La preocupación de reacciones adversas por uso de sueros y antisueros quedó plasmada en la descripción siguiente: En el uso de Seroterapia cada médico debe tener siempre presente este precepto fundamental claro y preciso: cada sujeto sometido a la Seroterapia puede estar en anafilaxia congénita o adquirida y reaccionar por lo tanto con enfermedad del suero. ${ }^{25}$

El uso de sangre completa del mismo individuo, la autohemoterapia, se popularizó en el período de los años 30's a los 60 's, para tratamiento de entidades como alcoholismo crónico: La técnica empleada por nosotros es la siguiente: tres veces por semana, inyección bajo la piel del vientre, de 10 a 12 c. c. de sangre del mismo paciente, retirada en el momento, y reinyectada sin ninguna preparación. Se ha pensado que la autohemoterapia actúa por el fenómeno de shock favorable en ciertas enfermedades infecciosas o hemorrágicas, o como un procedimiento de desensibilización en las dermatosis. En el caso particular que nos ocupa habría que pensar en una acción sensibilizante hacia el alcohol. Este punto, solamente podrá ser resuelto por medio de experiencias de laboratorio. ${ }^{26}$ También se usó en infecciones puerperales como la mastitis, ${ }^{27}$ entre otras enfermedades. Emulando lo que ahora conocemos como inmunomodulación, se ensayaron compuestos de oro para manejo de pacientes con tuberculosis en diferentes modalidades, similar a lo ocurrido posteriormente con su uso en artritis reumatoidea: El oro tiene una acción muy considerable en las formas ganglionares, óseas, cutáneas y serosas, en particular en las pleuresías crónicas. ${ }^{28}$

El impacto social planetario de mayor repercusión tuvo su mejor expresión en el uso de vacunas, con acciones complementarias de epidemiología y salud pública, matizando actividades estratégicas de prevención con los biológicos y proyecciones de educación para la salud. ${ }^{29}$ La vacunación contra la tuberculosis saltó a primera plana con la proposición de: vacunación antituberculosa propuesta y practicada desde hace varios años por Calmette para que se desarrolle en él la inmunidad contra la Tuberculosis provocada por el bacilo de Calmette- Guerin ${ }^{30,31} \ldots$ En el Hospital San Felipe se ha 
comenzado recientemente a aplicar el B. C. G. en el Servicio de Maternidad a los niños recién nacidos; esto en vía de ensayo, por supuesto, de acuerdo con la información que hemos recibido. ${ }^{32}$

La Inmunohematología cobró vida con la primera transfusión sanguínea: Corría el año de 1935. Sucedía que en el servicio de ginecología del Hospital San Felipe se encontraba una operada por embarazo ectópico roto con tremenda inundación peritoneal, quien por la hemorragia que había sufrido, se debatía entre la vida y la muerte... le hayan solicitado al Dr. Midence que, puesto que una transfusión ayudaría para rescatar de las garras de la muerte aquella paciente, la practicara una allí en el hospital y gentilmente y por principios profesionales haya accedido. ${ }^{33}$ Unos 15 años después se establecieron las bases para la creación del Banco de Sangre del Hospital General. ${ }^{29,30}{ }^{34,35}$ En albores del Servicio de Transfusión de la misma presentaron reacciones de tipo febril, precedidas de fuertes escalofríos $y$ tenemos que mencionar, con dolor, un caso fatal, debido a una infusión masiva de sangre citratada y suero fisiológico. Estas reacciones las atribuimos a la imperfecta esterilización de los frascos y juegos transfusores. ${ }^{36}$

El desarrollo combinado de diversas disciplinas afines a la Inmunología y la Infectología, tanto en el área básica (Genética, Bioquímica, Fisiología, otras), como clínica (Medicina Tropical, Patología, Parasitología, Micología, Dermatología, etc.), o Salud Pública (Epidemiología, Estadística, Administración, etc.), llevó siempre el trabajo amplio de eminentes médicos en la caracterización de gérmenes y su ambiente, el estudio de la respuesta del sistema inmunológico, y las aplicaciones pragmáticas para pacientes y sociedad. En el medio, merece particular mención el Dr. don Antonio Vidal Mayorga, entregado clínico, educador, gremialista, salubrista e investigador, que sumergido en el mundo microbiológico se preguntaba: ¿Cuál es la naturaleza de los ultravirus? ¿La ciencia ha logrado aislarlos del resto de los seres vivientes; pero ¿Qué son estos seres? ¿Qué forma afectan? ¿Son fluidos, son corpúsculos de otra cosa que nuestra imaginación no puede representarse ni concebir? Problema interesante, pero imposible de resolver en el estado actual de nuestros conocimientos. ${ }^{37}$ Su ardua labor en la caracterización de vectores transmisores de parásitos, especialmente el paludismo, lo llevó a describir especies de zancudos en diferentes áreas de la geografía nacional. ${ }^{38} \mathrm{En}$ comunicaciones diferentes, los doctores don Carlos A. Javier Santos y don Carlos A. Javier Zepeda, complementan un perfil de este connotado médico, aunque... Da la impresión de que no pudo realizar todo lo que él deseó, lo cual es explicable, pues su numen de sabio y artista debió sentirse comprimido por las mezquindades del medio y del momento histórico en que le tocó luchar. Sus escritos revelan una ilustración variada y exquisita, propia de un hombre que ha leído mucho, que ha viajado mucho, que ha vivido intensamente. ${ }^{39}$ La malaria, la tuberculosis, enfermedades intestinales y las exantemáticas de la infancia y otros padecimientos, se convirtieron en problemas médico-sociales cuyo control se salía de las manos del médico en forma individual y se volvió necesario organizar los sistemas de atención de la salud pública. ${ }^{40}$ Un logro fundamental alcanzado por el Dr. Vidal Mayorga fue la creación del Laboratorio Central de Serología, con el apoyo técnico y financiero del Servicio Cooperativo Interamericano de Salud Pública (SCISP), que en 1957 pasó a depender del Ministerio de Salud Pública y Bienestar Social. "Vidal fue un hombre fuera de su época..., dedicando su esfuerzo para tratar de organizar el sistema de salud pública..., su formación completa lo convirtió en un verdadero Patólogo; se desempeñaba en el laboratorio, en la clínica y en funciones administrativas. Fue además un admirado profesor de la Facultad de Medicina. ${ }^{3,4}$

Vidal Mayorga razonaba... "Una palabra, el médico debe ser biólogo. Debe interesarle el estudio de todo lo que vive y muere. La primera condición que todo Médico debe reunir para estar capacitado a dirigir su visión sobre horizontes más amplios y luminosos será una comprensión más rápida de los fenómenos naturales y una cultura más amplia y general. Mientras que la anafilaxia fue explicada como un fenómeno químico aislado, su importancia fue casi nula, pero desde el momento en que fue interpretada como un hecho general biológico, utilísimas fueron las deducciones y grandes los adelantos. El chocanafiláctico se redujo a una falta de equilibrio de los coloides protoplasmáticos y como bien sabéis que el protoplasma es la base de todo ser viviente (Huxley), resultó de esto una aplicación más vasta, y más general. Las bacterias mismas son susceptibles de experimentar el choc-anafiláctico... El asma esencial no existe, se trata de una lesión orgánica de un simple fenómeno de anafilaxia...que puede ser combatida por la vacunación antianafiláctica... y se obtiene la desensibilización empleando dosis mínimas repetidas de cada una de estas sustancias según el caso... Finalmente, cuando la causa es completamente desconocida, recurriremos a la administración de peptonas, a la autoseroterapia, autohemoterapia, y a la administración del calcio por la vía endovenosa" (sic). ${ }^{41}$

En los tiempos posteriores a la segunda guerra mundial surgen en el escenario clínico dos figuras médicas insignes, el dermatólogo don Hernán Corrales Padilla y el cirujano otorrinolaringólogo don Enrique Aguilar- Paz Cerrato. "llustres ciudadanos, maestros de muchas generaciones médicas de la Facultad de Medicina de la UNAH, clínicos finos, investigadores originales, apasionados gremialistas, políticos y funcionarios públicos probos, escritores prolíficos y hombres polifacéticos, que han dejado un enorme legado a la medicina local". ${ }^{42}$ Corrales Padilla incursionó intensamente en la descripción y manejo de patología infecciosa, alergias, autoinmunidad y neoplasia. A guisa de ejemplo, se exponen algunas referencias en sesudos escritos sobre fotodaño y fotobiología, ${ }^{43}$ autoinmunidad, ${ }^{44,45}$ incluyendo su ensayo sobre vitiligo, que reúne la experiencia clínica de muchos años de investigación en torno a esta enfermedad; ${ }^{46}$ enfermedades infecciosas ${ }^{47,48}$ y cáncer. ${ }^{49} \mathrm{El}$ mismo colega Aguilar Paz plasmó sus elogios al excelso maestro, como fuente de sabiduría e inspiración. ${ }^{50}$ En tanto que el dermatólogo don Héctor Laínez, con beca del gobierno de Inglaterra realizó estudios de Inmunología y Alergia en la Universidad de Londres (1965-1966), convirtiéndose en el primer inmuno- alergólogo del país. ${ }^{51}$ Aguilar Paz, de su parte, nos ha dejado grandes aportes que han facilitado la evolución de nuestras especialidades; aparte de su enorme producción 
de escritos médicos (ver una selección en un número dedicado a la otorrinolaringología), ${ }^{52}$ políticos y sociales, novelas costumbristas y poesía; reformó el sistema de salud del país y fundó el Hospital Escuela, centro por excelencia de desarrollo de múltiples especialidades: El post-grado de la Escuela de Medicina, para registro de la Historia, se gestó en el Ministerio de Salud, en nuestra gestión administrativa. ${ }^{53}$ "EI desarrollo ascendente y de gran acaecimiento de los posgrados de medicina al seno del HE [Hospital Escuela], a partir de los años 70 's, significó un gran impulso a la formación de talento humano, la investigación y la difusión del conocimiento". ${ }^{54}$

En la década de los 70's arribó un contingente de médicos que habían salido a especializarse al extranjero, como figuras que comenzaron a moldear un encuentro más estrecho entre ambas especialidades. Destacadas figuras como el Dr. don Carlos E. Gutiérrez G., primera figura reconocida en la especialidad de Alergia e Inmunología Clínica, y el Dr. don Carlos A. Javier Zepeda, patólogo y microbiólogo, quienes, actuando primariamente desde el seno del Departamento de Patología del Hospital San Felipe, lograron establecer los Servicios de Inmunología y de Microbiología, respectivamente, en el Departamento de Laboratorios Clínicos del HE, independiente del Departamento de Patología. ${ }^{55}$ Gutiérrez instauró pruebas inmunológicas de diagnóstico en sangre, suero o tejidos, tales como inmunofluorescencia, inmunodifusión, aglutinación, fijación de complemento o ELISA, entre otros. ${ }^{56}$ En tanto que Javier Zepeda se sumergió en una carrera de entrega a la asistencia y la docencia, y de profunda investigación, tal como lo atestigua su formidable producción de artículos científicos publicados preferentemente en la $\mathrm{RMH}$, pero también en revistas internacionales y otras nacionales. ${ }^{57} \mathrm{Los}$ primeros infectólogos, los Dres. Renato Valenzuela ${ }^{58}$ (Pediatría) y Milton González (medicina interna), llegaron a finales de la década, facilitando la fundación de la Sociedad Hondureña de Enfermedades Infecciosas (1982), nutrida por la generación de los 80 's, doctores Tito Alvarado, Elsa Palou, Efraín Bu Figueroa, Denis Padgett y Maribel Rivera. ${ }^{2}$ Los laboratorios clínicos evolucionaban a la modernización, más allá de lo que se conocen como las pruebas de rutina, con la aparición de las pruebas por inmunoensayos enzimáticos, cromatográficos y posteriormente de ácidos nucleicos, ampliando el apoyo al diagnóstico e intervenciones terapéuticas con técnicas inmunológicas y genéticas (plasmáticas, tisulares, celulares y moleculares), que permitieron la tipificación de gérmenes y otros determinantes antigénicos para diversas enfermedades y la producción de antisueros.

A mediados de los 80 's y 90 's ingresa un fuerte contingente de especialistas entre alergólogos, inmunólogos, infectólogos, epidemiólogos, microbiólogos, patólogos, parasitólogos, biólogos moleculares, que rápidamente se vieron involucrados en el desarrollo de modelos de trabajo en sida, tuberculosis, malaria, dengue, cólera, enfermedad de Chagas, otros parásitos (enfermedades desatendidas), micosis, control de infecciones y vacunas. El Dr. don Tito Alvarado Matute ${ }^{59}$ describió el primer caso de sida en el país en 1985, dando paso a una intensa respuesta de trabajo mancomunado entre clínicos, epidemiólogos, gerentes e investigadores, debido a la grave evolución epidémica. El sida vino a poner en crisis el sistema nacional de salud, desde ya frágil e insuficiente en atenciones a otras tareas como la mortalidad materno-infantil y epidemias de enfermedades transmisibles y no transmisibles agudas y crónicas (desnutrición, tuberculosis, dengue, etc.). En 1986 surge la Sociedad Hondureña de Alergia e Inmunología Clínica ${ }^{60}$ y se impulsan paralelamente las Asociaciones Centroamericanas de ambas especialidades. Unos años después (1993) se funda el Instituto de enfermedades Infecciosas y Parasitología Antonio Vidal (IAV). ${ }^{61}$ Alvarado se adelanta a hacer una revisión de la situación mundial del sida ${ }^{62}$ y exhorta a la autoridad sanitaria y la comunidad científica a iniciar la lucha de lo que se avizoraba como una catástrofe epidémica mundial y local. ${ }^{63}$ Para los primeros 10 años de la epidemia, Honduras daba cuenta de más del $60 \%$ de los casos de sida en Centroamérica, con una epidemia de carácter bimodal, i.e., generalizada en el corredor norte y concentrada en el resto del país. Rápidamente, se caracterizó el comportamiento de la infección por $\mathrm{VIH}$ y de los conglomerados sociales con mayor o menor riesgo, dando paso a una respuesta robusta con el respaldo internacional ${ }^{64} \mathrm{La}$ realidad es que el tema $\mathrm{VIH} /$ sida vino a nuclear el interés de la medicina clínica en este y otros tópicos de ambas especialidades, como lo testimonian los más de 100 artículos publicados en las revistas médicas nacionales en los últimos 25 años. Es así que se renovaron los intereses de la clínica en temas como el resto de las infecciones de transmisión sexual, ${ }^{65,66}$ tuberculosis, ${ }^{67-70}$ parasitosis, ${ }^{71-73}$ otras virosis, ${ }^{74-81} \mathrm{y}$ se afinaron técnicas de ácidos nucleicos en el diagnóstico. ${ }^{82,83}$

Otra figura egregia de nuestro medio es el Dr. don Salvador Moncada, quien sin duda alguna es el más brillante investigador que ha producido el país; su labor puede resumirse en la propia expresión de su pensamiento: Voy a referirme a mi trabajo científico de los últimos 20 años. Pero no a mi trabajo en general, sino a las situaciones en las que puedo discernir el proceso de descubrimiento. Son estos pocos momentos misteriosos que se resuelven en esa incomparable sensación de maravilla que es el descubrimiento científico, los que alcanzan para abastecer de esperanza la duda existencial que nos domina. Voy, además, a referirme a la ciencia experimental llevada a cabo no como consecuencia de inmensa tecnología, que es el signo de nuestra época, sino como resultado del uso de la curiosidad y del ingenio aplicados a la más sencilla de todas las técnicas de la biología experimental: el ensayo biológico. En 1971 tuve el privilegio de incorporarme al grupo dirigido por John Vane en el Departamento de Farmacología del Real Colegio de Cirujanos. Los frutos de mi trabajo de este período demostraron que, tanto en inflamación como en dolor las prostaglandinas no actúan como mediadores de ninguno de los signos o síntomas sino como moduladores, proporcionando un sistema de amplificación el que es reducido por fármacos del tipo de la aspirina... esto condujo a su empleo en situaciones nuevas tales como la prevención del parto prematuro, la estimulación del cierre del ductus arteriosus persistente y el tratamiento del síndrome de Bartter...los últimos veinte años han sido inmensamente estimulantes en relación a lo que denominó "casualidad, diseño, casualidad". Hemos tropezado 
con hallazgos que han constituido la base de desarrollos lógicos, sólo para volver a tropezar con lo inesperado. Si alguna guía he observado, ha sido la de mantener una visión amplia mientras analizamos sistemáticamente las hipótesis que constantemente lanzamos como redes. Así, hemos obtenido información acerca de una diminuta parcela de realidad previamente desconocida. EI rasgo más distintivo de todo este proceso ha sido la interacción humana y la manera en la cual tanta gente ha contribuido en forma positiva. ${ }^{84}$ El trabajo minucioso del equipo del laureado nobel J. Vane llevó al descubrimiento del mecanismo de acción de la aspirina y fármacos relacionados y su acción antitrombótica, diferentes prostaglandinas y sus consecuencias, del factor relajante de origen endotelial (EDRF) al óxido nítrico en el sistema vascular, el sistema nervioso y el sistema inmunitario, llevando, entre otros aspectos, al descubrimiento de drogas de acción en el sistema cardiovascular como el sildenafil. Sus aportes a la ciencia desde la plataforma de la Farmacología le han converido en un científico de dimensiones universales, proyectándose en el cultivo de nuevos científicos en su ambiente amplio de desarrollo, con gran énfasis en el ámbito nacional, a través de la academia. Fue galardonado con el premio "Príncipe de Asturias de Ciencia y Tecnología", postulado al premio Nobel en 1998 y declarado Sir por la Reina Isabel II en el 2010.

Desde la década de los años 70 el enorme progreso en la salud se vio reflejado en la vacunación. "En 1979 se creó oficialmente el Programa Ampliado de Inmunizaciones (PAI) de la Secretaria de Salud, con el objetivo de disminuir la morbilidad y mortalidad por enfermedades prevenibles por vacunación, a través de la vacunación de la población menor de cinco años, con énfasis en la menor de dos años, mujeres en edad fértil y grupos en riesgo para lograr el control, eliminación y erradicación de dichas enfermedades. Desde su creación se ha establecido como un programa prioritario de la política de salud, cuya estrategia básica es la oferta de servicios de vacunación gratuitos y permanentes en todos los niveles de la red de servicios de salud; lográndose en la última década notables avances, en materia de inmunización, ampliándose el acceso a los servicios de vacunación a más del $90 \%$ de la población infantil y mujeres en edad fértil, disminuyendo la incidencia y mortalidad por enfermedades prevenibles por vacunación, contribuyendo significativamente al descenso de la mortalidad infantil". ${ }^{85}$ La participación de miembros de la Sociedad Hondureña de Enfermedades Infecciosas en el PAI ha sido significativa tanto en asesoría como en monitoria y evaluación programática y de respuesta inmune a vacunas. ${ }^{86-89}$

La promoción de la lactancia materna ha sido un elemento relevante en la práctica clínica pediátrica, de medicina general, salud pública y otras especialidades, que también ha recalado a inmunoalergólogos e infectólogos, habida cuenta de su capital presencia en los programas de supervivencia infantil. ${ }^{90} \mathrm{El}$ manejo de las alergias alimentarias de la infancia ha reforzado su desarrollo, al tiempo que los alergólogos y pediatras han manejado los derivados lácteos proteicos hidrolizados, la exclusión láctea o la inducción de tolerancia y la regulación del microbioma intestinal, como formas de tratamiento a estas dolencias. ${ }^{91}$
Como los laboratorios clínicos y de patología han sido un punto de encuentro idóneo de ambas especialidades, respecto al diagnóstico y manejo de las diversas entidades clínicas, el aporte al trabajo de lucha contra las grandes epidemias desde esa perspectiva ha sido el de la caracterización fenotípica de las diferentes formas clínicas de presentación causadas por el germen. Por ejemplo, en las enfermedades parasitarias, bacterianas, micóticas y virales sistémicas como malaria, enfermedad de Chagas, tuberculosis, las virosis epidémicas como dengue, zika y chikungunya, el inmunodiagnóstico serológico o las pruebas in situ han sido esenciales. Los ensayos de inmunoadsorción enzimática son ahora de uso extendido para tal fin, al igual que los noveles ensayos de ácidos nucleicos, que, junto a la microbiología, han brindado una instrumentación amplia para el diagnóstico certero. En malaria, arbovirosis y Chagas, por ejemplo, en el laboratorio de Parasitología del Hospital Escuela se ha contribuido con el desarrollo de tales técnicas, comenzando con pruebas de tinción convencionales, pasando por pruebas rápidas serológicas y ensayos de biología molecular (reacción en cadena de polimerasa), lo que ha servido tanto para apoyar los programas de salud pública como para resolver casos no convencionales y graves. ${ }^{92-95} \mathrm{El}$ Departamento de Microbiología de la UNAH, fundado por el emérito doctor don Edmundo Poujol y potenciado con figuras científicas como Humberto Cosenza, Ivette Lorenzana y Annabelle Ferrera, ha dado un fuerte sustento al desarrollo de estas especialidades, estableciendo laboratorios modernos para la formación de estudiantes y apoyo a programas de salud pública. ${ }^{84,96}$ Carlos y Elisa Ponce desde el Laboratorio del Programa Nacional de Chagas hicieron sustantivos progresos para el diagnóstico, manejo y control de la enfermedad de Chagas y leishmaniasis, entre otros, el desarrollo de pruebas serológicas para diagnóstico clínico y de poblaciones ${ }^{97,98}$ En 1991 junto al grupo de Dermatología liderado por Corrales Padilla, describieron una variante clínica de leishmaniasis cutánea (The Lancet, 1991). Rina G. de Kaminsky ha sido otro baluarte que ha potenciado esta convergencia, como científica, académica, exdirectora del IAV y colaboradora en las actividades de la SHEI, en el año 2000 cofundó la Asociación Hondureña de Parasitología. ${ }^{97}$

La Inmunohematología se inició con la puesta en marcha del primer banco de sangre en el Hospital General; su modernización ocurrió a partir de los años 70 de la mano del Dr. don Salomón Grinspan ${ }^{99}$ y de la Dra. doña Elizabeth Vinelli en Cruz Roja Hondureña. ${ }^{100} \mathrm{La}$ seguridad transfusional se amplió con la adopción de la donación voluntaria o de reposición, no remunerada, y las pruebas serológicas para Chagas, ${ }^{101}$ hepatitis $B$, sifilis, VIH ${ }^{102}$ y luego hepatitis C y HTLV I/II. Actualmente la Cruz Roja Hondureña maneja las pruebas moleculares (NAT) para VIH y hepatitis.

El interés por manejar adecuadamente los accidentes por mordeduras de serpientes se ha mantenido como parte de la práctica médica, ${ }^{103-105}$ pero ha cobrado particular interés por el impulso que se le ha imprimido en los últimos años, ${ }^{94}$ movilizando recursos, promocionando la enseñanza y estableciendo líneas de cooperación con el Instituto Clodomiro Picado de Costa Rica. La eclosión de un nuevo coronavirus, Sars-CoV-2, que 
ha provocado la inesperada pandemia de covid- $19,{ }^{107}$ ha puesto en jaque la salud y la economía mundiales y, por supuesto la nacional, generando retos extraordinarios para la medicina del país, conjuntando diferentes especialidades, incluyendo Infectología e Inmunología, con impulsos impresionantes para salvaguardar la salud pública y de las personas. Esos esfuerzos se han traducido en la labor denodada de clínicos atendiendo pacientes con la enfermedad, la emisión de directrices técnicas para la toma de decisiones, formulación de plataformas para la vigilancia epidemiológica, la educación en salud para la protección personal y la búsqueda innovadora de tratamientos eficaces para reducir la carga viral y sus consecuencias. ${ }^{108}$

En conclusión, en el devenir histórico de la medicina de Honduras (y el mundo), el desarrollo de las especialidades de Inmunología e Infectología ha sido paralelo y combinado, habida cuenta de la estrecha vinculación entre los gérmenes y el sistema de respuesta, interacción (microbioma-respuesta inmunitaria) ambientada en el contexto inmunogenético del individuo y el "exposoma", originando diversas patologías que conllevan disregulaciones del sistema inmunitario que a su vez condicionan diversas entidades nosológicas (infección, autoinmunidad, alergia, inmunodeficiencia, degeneración 0 neoplasia), a las que se ve enfrentado el médico a lo largo de la evolución de la sociedad. Esta narración ha privilegiado una pesquisa de coevolución de ambas especialidades en el ámbito nacional, a partir esencialmente de información científica encontrada en publicaciones locales, sustancialmente la RMH, como instrumento principal de la difusión del conocimiento de la medicina en el país. ${ }^{109}$

\section{REFERENCIAS}

1. Fernández JA. Inmunología en la prevención. Rev Med Hondur [Internet]. 1990 [acceso 2 de febrero 2020];58(2):130-132. Disponible en http://www. bvs.hn/RMH/pdf/1990/pdf/Vol58-2-1990-7.pdf

2. Javier Zepeda CA. Sinopsis de la evolución de la infectología en Honduras. Rev Méd Hondur [Internet]. 2011 [acceso 2 de febrero 2020]; 79(3): 146152. Disponible en: http://www.bvs.hn/RMH/pdf/2011/pdf/Vol79-3-2011-10. pdf

3. Espinoza Murra D, Carías SA, Gálvez LC, Valenzuela Castillo R. Enseñanza y práctica de la medicina: reseña histórica de la Facultad de Ciencias Médicas de la UNAH. Rev Fac Cienc Méd [Internet]. 2010 [acceso 2 de febrero 2020];7(supl 2):7-50. Disponible en: http://cidbimena. desastres.hn/RFCM/pdf/2010/pdf/RFCMVol7-S2-2010-3.pdf

4. Javier Zepeda CA. Reseña histórica de la Revista Médica Hondureña 1930-2005. Rev Med Hondur [Internet]. 2005[acceso 2 de febrero 2020]; 73(Supl 2):4-16. Disponible en http://www.bvs.hn/RMH/pdf/2005/pdf/ Vol73-S2-2005-3.pdf

5. Bu Figueroa E. La página editorial, expresión del pensamiento médico: algunos rasgos históricos. Rev Med Hondur [Internet].2000[Acceso 2 de febrero de 2020]; (ed. Esp.): 5-12. Disponible en: http://www.bvs.hn/RMH/ pdf/2000/pdf/espcom-2000-2.pdf

6. Rivera Williams C. Historia de la medicina en Honduras. Tegucigalpa: Graficentro Editores; 2007.

7. Castellanos Delgado PE. Buscando raíces a través de la historia de la medicina. Tegucigalpa: Litografía López; 2002.

8. Dávila B. Historia de la medicina en Honduras. [Internet]. Tegucigalpa: UNAH, Facultad de Ciencias Médicas, Departamento de Medicina Preventiva y Social; s.f. [acceso 2 de febrero 2020]. Disponible en http:/l www.bvs.hn/Honduras/Historia/HistoriaMedicinaHondurena.pdf

9. Calderón A. Historia de la medicina en Honduras. [Internet]. [Acceso 2 de febrero de 2020]. Disponible en https://prezi.com/mzyssd4qjcik/historiade-la-medicina-en-honduras/

10. Bourdeth Tosta JA. El Cólera en la Historia de Honduras Rev Med Hondur [Internet]. 1995 [Acceso 2 de febrero 2020];63(2):83-85. Disponible en http://www.bvs.hn/RMH/pdf/1995/pdf/Vol63-2-1995-13.pdf

11. Historia de la Facultad de Ciencias Médicas. [Internet]. Presentación s/f. Tegucigalpa. [Acceso 2 de febrero de 2020]. Disponible en http://www.bvs. $\mathrm{hn} /$ Honduras/Historia/HistoriaFFCCMM.pdf

12. Historia de la Medicina. Biografías. August Paul von Wasserman. [Internet]. Acceso 2 de febrero de 2020. Disponible en https://www. historiadelamedicina.org/wassermann.html

13. Diagnóstico biológico de la tuberculosis. Rev Med Hondur [Internet]. 1931 [acceso 2 de febrero 2020]; 2(18):58. Disponible en: http://www.bvs.hn/ $\mathrm{RMH} / \mathrm{pdf} / 1931 / \mathrm{pdf} / \mathrm{A} 1-9-1931-13 . \mathrm{pdf}$

14. La prueba Aschheim -Zondek y su aplicación a la diagnosis de preñez, epitelionia corionico y mola hidatidiforme. Rev Méd Hondur [Internet]. 1931 [Acceso 2 de febrero 2020]; 2(18): 53-57. Disponible en: http://www.bvs. $\mathrm{hn} / \mathrm{RMH} / \mathrm{pdf} / 1931 / \mathrm{pdf} / \mathrm{A} 1-9-1931-12 . \mathrm{pdf}$

15. Vidal A. Las nuevas adquisiciones serológicas en el diagnóstico de la Sífilis. Rev Med Hondur [Internet]. 1931 [Acceso 2 de febrero 2020]; 1 (11):3-9.Disponible en: http://www.bvs.hn/RMH/pdf/1931/pdf/A1-11-19312.pdf

16. Cáceres Vigil M. Paludismo y Serorreacción de Kahn. Rev Med Hondur [Internet]. 1934 [acceso 2 de febrero 2020]; 4(1):153-157. Disponible en: http://www.bvs.hn/RMH/pdf/1934/pdf/A4-1-1934-10.pdf

17. Jaubert A, Goy P. Contribución al estudio de la vacunoterapia de las infecciones de origen gonocócico. Rev Med Hondur [Internet]. 1932 [acceso 2 de febrero 2020]; 2(1):4-21. Disponible en: http://www.bvs.hn/ $\mathrm{RMH} / \mathrm{pdf} / 1932 / \mathrm{pdf} / \mathrm{A2}-1-1932-2 . \mathrm{pdf}$

18. Montoya Álvarez J. Transfusión de plasma de sangre con reacciones serológicas positivas por sífilis. Resúmenes de literatura hasta el presente. Rev Med Hondur [Internet]. 1951[acceso 2 de febrero 2020]; 21 (2):335336. Disponible en: http://www.bvs.hn/RMH/pdf/1951/pdf/A21-2-1951-4. pdf

19. González Galván JM. La Sueroterapia Sistemática en la Apendicitis Aguda. Rev Med Hondur [Internet]. 1932 [acceso 2 de febrero 2020]; 2(3):3-5. Disponible en: http://www.bvs.hn/RMH/pdf/1932/pdf/A2-3-1932-2.pdf

20. Nuevas experiencias demostrativas de la curación de la difteria en el cobayo por la Haptinogenina [Méndez], y comparación con la obtenida por el suero antidiftérico. Rev Med Hondur [Internet]. 1932 [acceso 2 de febrero 2020]; 2(5): 60-63. Disponible en: http://www.bvs.hn/RMH/ pdf/1932/pdf/A2-5-1932.pdf

21. La lucha contra el sarampión utilizando el suero de convaleciente. Rev Med Hondur [Internet]. 1932 [acceso 2 de febrero 2020]; 2(8):13-17. Disponible en: http://www.bvs.hn/RMH/pdf/1932/pdf/A2-8-1932-3.pdf

22. Stroud WD, Goldsmith MA, Polk DS, Thorp FQ. Ten years' observation of children with rheumatic heart disease. JAMA 1933; 101: 502.

23. Suarez G. Las nuevas ideas sobre la etiología, evolución y tratamiento de la enfermedad reumática. Rev Med Hondur [Internet]. 1934 [acceso 2 de febrero 2020]; 4(5): 432-436. Disponible en: http://www.bvs.hn/RMH/ pdf/1934/pdf/A4-5-1934-17.pdf

24. Selva E, Aguilar R. El Antiveneno botrófico en las diátesis hemorrágicas. Rev Med Hondur [Internet]. 1936 [acceso 2 de febrero 2020]; 6(1):101102. Disponible en: http://www.bvs.hn/RMH/pdf/1936/pdf/A6-1-1936-6.pdf

25. Martinico J. Reproducción de artículo por Martinico J. La enfermedad de suero. Rev Med Hondur [Internet]. 1934 [acceso 2 de febrero 2020]; 4(4): 338. Disponible en: http://www.bvs.hn/RMH/pdf/1934/pdf/A4-4-1934-5.pdf

26. La autohemoterapia. Tratamiento muy eficaz del alcoholismo inveterado. Rev Med Hondur [Internet]. 1932 [acceso 2 de febrero 2020]; 2(6): 58-62. Disponible en: http://www.bvs.hn/RMH/pdf/1932/pdf/A2-6-1932-9.pdf

27. Paredes S. Reproducción de artículo por de Breslau LS. Tratamiento de las mastitis puerperales por autohemoterapia. Rev Med Hondur [Internet]. 1936 [acceso 2 de febrero de 2020]; 6(4):294. Disponible en: http://www. 
bvs.hn/RMH/pdf/1936/pdf/A6-4-1936-8.pdf

28. Auroterapia. La práctica de la auroterapia en los tuberculosos. Rev Med Hondur [Internet]. 1931 [acceso 2 de febrero 2020]; 1(11): 39-41. Disponible en: http://www.bvs.hn/RMH/pdf/1931/pdf/A1-11-1931-12.pdf

29. [Secretaría de la Liga de Sociedades de la Cruz Roja, París]. La Lucha contra las Enfermedades Epidémicas. Rev Med Hondur [Internet]. 1936 [acceso 2 de febrero de 2020]; 6(3):229-232. Disponible en: http://www. bvs.hn/RMH/pdf/1936/pdf/A6-3-1936-11.pdf

30. Morera Carbonell J. Conceptos generales sobre profilaxis y servicios Antituberculosos. Rev Med Hondur [Internet]. 1937 [acceso 2 de febrero 2020]; 7(5): 358-378. Disponible en: http://www.bvs.hn/RMH/pdf/1937/pdf/A7-51937-10.pdf

31. Domingo P. Reproducción de artículo por Domingo P. Síntesis de los fundamentos científicos y de la práctica de la vacunación antituberculosa por el B. C. G. Rev Med Hondur [Internet]. 1947 [acceso 2 de febrero 2020]; 17(1): 375-383. Disponible en: http://www.bvs.hn/RMH/pdf/1947/ pdf/A17-1-1947-8.pdf

32. Díaz H. La vacunación por el B. C. G. Rev Med Hondur [Internet]. 1952 [acceso 2 de febrero de 2020]; 20(2): 157-158. Disponible en: http://www. bvs.hn/RMH/pdf/1952/pdf/Vol20-4-1952-1.pdf

33. Matute Canizales E. Como y cuando se hizo la Primera transfusión sanguínea. [Internet]. [Acceso 2 de febrero de 2020]. Disponible en http:// www.bvs.hn/Honduras/Historia/TransfucionSanguinea.pdf.

34. Bermúdez A. Necesitamos un Banco de Sangre. Rev Med Hondur [Internet]. 1946 [acceso 2 de febrero de 2020]; 16 (6): 303-306. Disponible en: http://www.bvs.hn/RMH/pdf/1946/pdf/A16-6-1946-4.pdf

35. Midence AC. Plática sobre la importancia del Banco de Sangre y la inocuidad de las transfusiones. Rev Med Hondur [Internet]. 1951 [Acceso 2 de febrero 2020]; 21(4): 413-418. Disponible en: http://www.bvs.hn/RMH/ $\mathrm{pdf} / 1951 / \mathrm{pdf} / \mathrm{A21}-4-1951-2 . \mathrm{pdf}$

36. Fiallos FJ. Reacciones adversas asociadas a la administración de sangre total. Derivados sanguíneos, soluciones cristaloides y material similar. Rev Med Hondur [Internet]. 1951 [acceso 2 de febrero 2020]; 21(6): 8-17. Disponible en: http://www.bvs.hn/RMH/pdf/1951/pdf/A21-6-1951-2.pdf

37. Vidal A. Los ultravirus y las formas filtrantes de los microbios visibles. Rev Med Hondur [Internet]. 1934 [acceso 2 de febrero 2020]; 4(3): 259-262. Disponible en: http://www.bvs.hn/RMH/pdf/1934/pdf/A4-3-1934-2.pdf

38. Vidal A. Zancudos Anofeles de Honduras. Rev Med Hondur [Internet]. 1944 [acceso 2 de febrero de 2020]; 14(3): 439-458. Disponible en: http:// www.bvs.hn/RMH/pdf/1944/pdf/A14-3-1944-2.pdf

39. Javier CA. Dr. Antonio Vidal M. Un precursor infatigable y panorámico del progreso de la medicina nacional. Rev Med Hondur [Internet]. 1978 [acceso 2 de febrero de 2020]; 46(3): 91-95. Disponible en: http://www.bvs. $\mathrm{hn} / \mathrm{RMH} / \mathrm{pdf} / 1978 / \mathrm{pdf} /$ Vol46-3-1978-10.pdf

40. Castellanos P. Evolución histórica de la Salud en Honduras durante el siglo XX. Rev Med Hondur [Internet]. 2005 [Acceso 2 de febrero 2020]; 73(Supl 2):17-36. Disponible en: http://www.bvs.hn/RMH/pdf/2005/pdf/ Vol73-S2-2005-4.pdf

41. Vidal A. Al margen de nuestros conocimientos Médico-Quirúrgicos. Rev Med Hondur [Internet]. 1936 [acceso 2 de febrero 2020]; 6(1): 96-101. Disponible en: http://www.bvs.hn/RMH/pdf/1936/pdf/A6-1-1936-6.pdf

42. Bourdeth Tosta JA. Médicos Destacados en Honduras. Rev Med Hondur [Internet]. 1998 [acceso 2 de febrero 2020]; 66(4): 171-178. Disponible en: http://www.bvs.hn/RMH/pdf/1998/pdf/Vol66-4-1998-9.pdf

43. Corrales Padilla H. Fotodermatosis. Introducción. Rev Med Hondur [Internet]. 1973 [acceso 2 de febrero de 2020]; 41(2):104-108. Disponible en: http://www.bvs.hn/RMH/pdf/1973/pdf/Vol41-2-1973.pdf

44. Corrales Padilla H. Dermatología en el Trópico Centroamericano. II Penfigo foliáceo. Rev Med Hondur [Internet]. 1966 [acceso 2 de febrero 2020]; 34 (1): 50-59. Disponible en: http://www.bvs.hn/RMH/pdf/1966/pdf/ Vol34-1-1966-8.pdf

45. Corrales Padilla H. Fotoquimioterapia de la Psoriasis con Psoralenes por vía oral y luz negra. Rev Med Hondur [Internet]. 1976 [acceso 2 de febrero de 2020]; 44 (2): 96-105. Disponible en: http://www.bvs.hn/RMH/pdf/1976/ $\mathrm{pdf} /$ Vol44-2-1976-5.pdf

46. Martel B, Bu Figueroa E. Grandes figuras de la medicina hondureña. El Doctor Hernán Corrales Padilla. Rev Med Hondur [Internet] 1996 [no hay fecha de cita]; 64(3): 116-117. http://www.bvs.hn/RMH/pdf/1996/pdf/Vol643-1996-11.pdf

47. Corrales Padilla H. Micosis profundas en Honduras. Rev Med Hondur [Internet]. 1955 [acceso de febrero de 2020]; 23(4):1019-1085. Disponible en: http://www.bvs.hn/RMH/pdf/1955/pdf/Vol23-4-1955.pdf

48. Corrales Padilla $\mathrm{H}$, Laínez $\mathrm{H}$. Leishmaniasis cutánea diseminada. Rev Med Hondur [Internet].1968 [acceso 2 de febrero 2020]; 36(2): 142-148. Disponible en: http://www.bvs.hn/RMH/pdf/1968/pdf/Vol36-2-1968-4.pdf

49. Corrales Padilla H. Carcinogénesis. Rev Med Hondur [Internet]. 1966 [acceso 2 de febrero 2020]; 34(3): 180-188. Disponible en: http://www.bvs. $\mathrm{hn} / \mathrm{RMH} / \mathrm{pdf} / 1966 / \mathrm{pdf} / \mathrm{Vol} 34-3-1966-4 . \mathrm{pdf}$

50. Aguilar Paz E. Homenaje al Maestro Hernán Corrales Padilla. Hernán, Inmortal. Rev Med Hondur [Internet]. 1999 [acceso 2 de febrero 2020]; 67(1): 125. Disponible en: http://www.bvs.hn/RMH/pdf/1999/pdf/Vol67-11999-16.pdf

51. Sandoval NJ. Precursores y pioneros de la dermatología en Honduras. Rev Med Hondur [Internet]. 2008 [acceso 2 de febrero 2020]; 76 (3):132137. Disponible en: http://www.bvs.hn/RMH/pdf/2008/pdf/Vol76-3-200811.pdf

52. Revista Médica Hondureña. Volumen 67, Número 1, Año 1999. [Internet]. 1999 [acceso 2 de febrero 2020]; 67 (1). Disponible en: http://www.bvs.hn/ $\mathrm{RMH} / \mathrm{pdf} / 1999 / \mathrm{htm} / /$ Vol67-1-1999.htm

53. Aguilar Paz E. El Hospital Escuela (un sueño, una realidad). Rev Med Hondur [Internet] 2005 [acceso 2 de febrero 2020]; 73 (Supl 2): S45-S50. Disponible en: http://www.bvs.hn/RMH/pdf/2005/pdf/Vol73-S2-2005-6.pdf

54. Vargas Pineda C, Matamoros M, Hakkert R. Los postgrados de medicina en Honduras: antecedentes, logros y metas. Rev Med Post UNAH [Internet] 1996 [acceso 2 de febrero 2020]; 1(1):49-56. Disponible en: http://www.bvs.hn/RMP/pdf/1996/pdf/Vol1-1-1996-11.pdf

55. Cardonal López V. Remembranzas de la especialidad de patología en Honduras de 1952 a 2005. Rev Med Hondur [Internet] 2011 [acceso 2 de febrero 2020]; 79 (1): 43-45. Disponible en: http://www.bvs.hn/RMH/ $\mathrm{pdf} / 2011 / \mathrm{pdf} /$ Vol79-1-2011-13.pdf

56. Zúniga GA, Biza S, Gutiérrez C, Durón RA. Cirrosis biliar primaria. Primer caso informado en la literatura hondureña. Rev Med Hondur [Internet] 1977 [acceso 2 de febrero 2020]; 45 (3-4): 99-103. Disponible en: http:// www.bvs.hn/RMH/pdf/1977/pdf/Vol45-3-4-1977-2.pdf

57. Fernández Vázquez J, Alger J. Reseña biográfica del Dr. Carlos A. Javier Zepeda Rev Med Hondur [Internet]. 2007 [acceso 2 de febrero 2020]; 75:102-106. Disponible en: http://cidbimena.desastres.hn/RMH/pdf/2007/ $\mathrm{pdf} /$ Vol75-2-2007-11.pdf

58. Fernández JA, Alger J, Bu Figueroa E. Renato Valenzuela Castillo: Una carrera para la academia ligada a la Infectología y la tecnología educacional en salud en Honduras. Rev Med Hondur [Internet]. 2011 [acceso 2 de febrero 2020]; 79 (3): 153-154. Disponible en: http://www. bvs.hn/RMH/pdf/2011/pdf/Vol79-3-2011-11.pdf

59. Fernández JA, Alger J. Tito Alvarado Matute: visión inspiradora de confraternidad. Rev Med Hondur [Internet]. 2013 [acceso 2 de febrero 2020]; 81(2-4): 125-127. Disponible en: http://www.bvs.hn/RMH/pdf/2013/ pdf/Nol81-2-4-2013-18.pdf

60. Reseña Histórica de la Creación de las Sociedades Médicas de Honduras. Rev Med Hondur [Internet]. 1987 [acceso 2 de febrero 2020]; 55(4): 352369. Disponible en: http://www.bvs.hn/RMH/pdf/1987/pdf/Vol55-4-1987-6. pdf

61. Instituto de Enfermedades Infecciosas y Parasitología Antonio Vidal. [acceso 2 de febrero 2020]; Disponible en: http://www.bvs.hn/php/level.ph p?lang=es\&component=35\&item $=3$, acceso 2 de febrero de 2020 .

62. Alvarado T. Síndrome de Inmunodeficiencia Adquirida, SIDA: consideraciones históricas, epidemiológicas, etiológicas, inmunológicas, clínicas, terapéuticas y de control. Rev Med Hondur [Internet]. 1985 [acceso 2 de febrero 2020]; 53 (3): 177-189. Disponible en: http://www. bvs.hn/RMH/pdf/1985/pdf/Vol53-3-1985-2.pdf

63. Alvarado T. SIDA: Un problema de salud Internacional y Nacional. Rev Med Hondur [Internet]. 1990 [acceso 2 de febrero 2020]; 58 (2): 105. Disponible en: http://www.bvs.hn/RMH/pdf/1990/pdf/Vol58-2-1990-1.pdf

64. Fernández JA. Cronología de la lucha contra el sida en Honduras. Rev Med Hondur. Próximo a publicar 2019; 87 (2). 
65. García Gámez R, Guevara Gutiérrez J. Asociación de HIV con condilomatosis genital en pacientes embarazadas, en el Hospital Materno Infantil durante 1998 y 1999. Rev Med Postgrados Med, UNAH [Internet]. 2001 [acceso 2 de febrero 2020]; 6 (3): 299-302. Disponible en: http://www. bvs.hn/RMP/pdf/2001/pdf/Vol6-3-2001-18.pdf

66. Urquía MA. Sobre el manejo sindrómico de infecciones de transmisión sexual. Rev Med Hondur [Internet]. 2005 [acceso 2 de febrero 2020]; 73(3): 104-105. Disponible en: http://www.bvs.hn/RMH/pdf/2005/pdf/ Vol73-3-2005-1.pdf

67. Romero Salandia M, Godoy Mejía C, Espinoza Alvarado I. Seroprevalencia de sífilis congénita en el Hospital Materno Infantil - Hospital Escuela. Rev Med Postgrados Med UNAH [Internet]. 1999 [acceso 2 de febrero 2020]; 4(3): 242-247. Disponible en: http://www.bvs.hn/RMP/pdf/1999/pdf/Vol4-31999-12.pdf

68. Díaz Flores A, Rivera DM, Medina M. Formas clínicas de tuberculosis en pacientes pediátricos atendidos en el Hospital Escuela, Bloque Materno Infantil 2001-2003. Rev Med Postgrados Med, UNAH [Internet]. 2006 [acceso 2 de febrero 2020]; 9 (1): 17-23. Disponible en: http://www.bvs.hn/ RMP/pdf/2006/pdf/Vol9-1-2006-5.pdf

69. Dubón JM, Rigouts L, Portaels F. Diagnóstico Serológico de Tuberculosis Utilización del antígeno P32 y el antígeno SLIV, para el diagnóstico rápido y precoz de la tuberculosis por la serología (ELISA) en el Hospital Regional IHSS en San Pedro Sula, Honduras. Rev Med Hondur [Internet]. 1993 [acceso 2 de febrero 2020]; 61(2): 45-50. Disponible en: http://www.bvs. hn/RMH/pdf/1993/pdf/Vol61-2-1993-4.pdf

70. Padgett Moncada D, Palou E. Estudio clínico retrospectivo de tuberculosis y SIDA en el Instituto Nacional del Torax, Tegucigalpa, Honduras. Rev Med Hondur [Internet]. 1993 [acceso 2 de febrero 2020]; 61(3):78-83. Disponible en http://www.bvs.hn/RMH/pdf/1993/pdf/Vol61-3-1993-2.pdf

71. Kaminsky RG. Estrongiloidiasis diseminada en una paciente viviendo con SIDA en Honduras. Rev Med Hondur [Internet]. 2005 [acceso 2 de febrero 2020]; 73(1): 34-39. Disponible en: http://www.bvs.hn/RMH/pdf/2005/pdf/ Vol73-1-2005-9.pdf

72. Kaminsky RG, Stovall ME, Mayer ML, Martin AD, Bowers LC, Didier ES. Microsporidia intestinales en pacientes viviendo con SIDA en Honduras. Rev Med Hondur [Internet]. 2007 [acceso 2 de febrero 2020]; 75(3): 116123. Disponible en: http://www.bvs.hn/RMH/pdf/2007/pdf/Vol75-3-2007-3. pdf

73. Sorto R, Bu Figueroa E. Perfil clínico-parasitológico de pacientes con VIH/ SIDA y diarrea crónica atendidos en el Hospital Escuela del 2003 al 2005. Rev Med Hondur [Internet]. 2006 [acceso 2 de febrero 2020]; 74(2): $69-76$. Disponible en: http://www.bvs.hn/RMH/pdf/2006/pdf/Vol74-2-2006-2.pdf

74. Lorenzana de Rivera I, Mourra S, de Gallo IG. Prevalencia de Anticuerpos Anti-HTLV-1 en comunidades de la Costa Norte de Honduras. Rev Med Hondur [Internet]. 1992 [acceso 2 de febrero 2020]; 60 (1): 13-17. Acceso 2 de febrero de 2020. Disponible en: http://www.bvs.hn/RMH/pdf/1992/pdf/ Vol60-1-1992-3.pdf

75. Grinspan S, Grave de Peralta L. Seroprevalencia de Hepatitis B y C en Donadores de Sangre. Rev Med Hondur [Internet]. 1993 [acceso 2 de febrero 2020]; 61(2): 42-44. Disponible en: http://www.bvs.hn/RMH/ pdf/1993/pdf/Vol61-2-1993-3.pdf

76. Lorenzana de Rivera I, Vinelli de Rivera E, Parham Aceituno L. Prevalencia de HTLV-I/HTLV-II en donantes de Sangre de la Cruz Roja Hondureña, determinado por PCR. Rev Med Hondur [Internet] 2004 [acceso 2 de febrero 2020]; 72 (1): 3-9. Disponible en: http://www.bvs.hn/RMH/pdf/2004/ $\mathrm{pdf} /$ Vol72-1-2004-2.pdf

77. Ambinder R, Brownig P, Lorenzana I, Levenihal B, Medina R, Cosenza H, et al. Epstein Barr virus y Enfermedad de Hodgkin en niños de Honduras y Estados Unidos de América. Hond. Ped [Internet]. 1993 [acceso 2 de febrero 2020]; 16 (1): 13-17. Acceso 2 de febrero de 2020. Disponible en: http://www.bvs.hn/RHP/pdf/1993/pdf/Vol16-1-1993-6.pdf

78. Ambinder R, Brownig P, Lorenzana I, Levenihal B, Medina R, Cosenza H, et al. Epstein Barr virus y Enfermedad de Hodgkin en niños de Honduras y Estados Unidos de América. Hond. Ped [Internet]. 1993 [acceso 2 de febrero 2020]; 16 (1): 13-17. Acceso 2 de febrero de 2020. Disponible en: http://www.bvs.hn/RHP/pdf/1993/pdf/Vol16-1-1993-6.pdf

79. Jovel López LE, Sánchez Colindres I. Infección por virus del papiloma humano (VPH) en niños infectados por el VIH. Acta pediátr. Hondur [Internet]. 2011 [acceso 2 de febrero 2020]; 2(1): 122. Disponible en: http:// www.bvs.hn/APH/pdf/APHVol2/pdf/APHVol2-1-2011-8.pdf

80. Alvarado Matute T. Hepatitis C: un cambio de paradigma. Rev Med Hondur [Internet]. 2014 [acceso 2 de febrero de 2020]; 82(2):81. Disponible en: http://www.bvs.hn/RMH/pdf/2014/pdf/Nol82-2-2014-11.pdf

81. Bu Figueroa E. Síndrome Respiratorio Agudo Severo (SARS). Rev Med Hondur [Internet]. 2003 [acceso 2 de febrero 2020]; 71(1):42-47. Disponible en: http://www.bvs.hn/RMH/pdf/2003/pdf/Vol71-1-2003-10.pdf

82. Lorenzana de Rivera I, Murillo Barahona W. Aplicación del PCR-ADN en el diagnóstico de la infección por VIH-1 en infantes. Rev Med Hondur [Internet]. 2003 [acceso 2 de febrero de 2020]; 71(3): 123-130. Disponible en: http://www.bvs.hn/RMH/pdf/2003/pdf/Vol71-3-2003-3.pdf

83. Hesse H, Medina MT, Lawrence D, Major EO, Bú-Figueroa E, Pavón R. Caracterización clínica y neuropsicológica de pacientes hondureños con demencia asociada al VIH-1 y su correlación con las moléculas inflamatorias en LCR y suero. Rev Med Postgrados Med, UNAH [Internet]. 2003 [acceso 2 de febrero 2020]; 8(1-2-3): 11-21. Disponible en: http:// www.bvs.hn/RMP/pdf/2003/pdf/Vol8-123-2003-4.pdf

84. Moncada S. Aventuras en farmacología: veinte años de casualidad y diseño. Rev Hondur Neurociencias [Internet] 1997 [acceso 2 de febrero de 2020]; 1:41-74. Disponible en: http://www.bvs.hn/RHN/pdf/1997/pdf/Vol11-1997-6.pdf

85. Molina IB. El impacto de las inmunizaciones en Honduras. En: Memorias IX Curso y VII Congreso Nacional de Pediatría Dr. Asdrubal Raudales Alvarado. Rev Hondur Pediatr. [Internet] 1999 [acceso 2 de febrero de 2020];20(3):104-105. Disponible en:http://www.bvs.hn/RHP/pdf/1999/pdf/ Vol20-3-1999-18.pdf

86. Ochoa N. Bu Figueroa E, Mejía MC. Respuesta inmune humoral a la vacuna antisarampionosa en niños menores de 5 Años. Rev Med Hondur [Internet]. 1991 [acceso 2 de febrero 2020]; 59(4): 175-178. Disponible en: http://www.bvs.hn/RMH/pdf/1991/pdf/Nol59-4-1991-3.pdf

87. Villatoro Godoy G, Rivera Medina M, Matamoros M, Palma F. Situación clínica epidemiológica de la tosferina en el Hospital Materno Infantil, Tegucigalpa, Honduras 1997. Revista Honduras Pediátrica [Internet]. 1998 [acceso 2 de febrero de 2020]; 19(4): 69-74. Disponible en: http://www.bvs. $\mathrm{hn} / \mathrm{RHP} / \mathrm{pdf} / 1998 / \mathrm{pdf} /$ Vol19-4-1998-3.pdf

88. García Jiménez S. Impacto de las Inmunizaciones en las Enfermedades Inmunoprevenibles en Centroamérica. Rev Hondur Pediatr [Internet]. 1999 [acceso 2 de febrero 2020]; 20(3): 75-76. Disponible en: http://www.bvs.hn/ RHP/pdf/1999/pdf/Vol20-3-1999-5.pdf

89. Banegas OG, Alemán G, Mejía N. Proyecciones del impacto con la introducción de la vacuna antineumococica en Honduras. Acta Pediatr Hondur [Internet]. 2011 [acceso 2 de febrero de 2020]; 2(1): 123-128. Disponible en: http://www.bvs.hn/APH/pdf/APHVol2/pdf/ APHVol2-1-2011-9.pdf

90. UNICEF. Grupo Lactancia Materna. La lactancia materna y el empleo de leche humana. Revista Honduras Pediátrica [Internet]. 1999 [acceso 2 de febrero 2020]; 20(4):219-221. Acceso 2 de febrero de 2020. Disponible en: http://www.bvs.hn/RHP/pdf/1999/pdf/Vol20-4-1999-7.pdf

91. Sorensen RU. Infecciones, vacunación y alergia. En:Memorias XXI Congreso Centroamericano y del Caribe de Pediatría V XII Curso Internacional de Pediatría. Rev Hondur Pediatr [Internet]. 2000 [acceso 2 de febrero de 2020];21(3):67. Disponible en: http://www.bvs.hn/RHP/ $\mathrm{pdf} / 2000 / \mathrm{pdf} / \mathrm{Vol} 21-3 \mathrm{E}-2000-16 . \mathrm{pdf}$

92. Fernández RD, García Y, Alger J. Malaria y Embarazo: Observaciones Clínico-Epidemiológicas en dos Zonas Geográficas de Honduras. Rev Med Hondur [Internet] 2001 [acceso 2 de febrero 2019]; 69(1): 8-18. Disponible en: http://www.bvs.hn/RMH/pdf/2001/pdf/Vol69-1-2001-3.pdf

93. Aguilar CJ, Bu Figueroa E, Alger J. Caracterización clínica y epidemiológica de la malaria en una comunidad endémica de Honduras. Rev Med Hond [Internet]. 2004 [acceso 2 de febrero de 2020]; 72:179-186. Disponible en: http://www.bvs.hn/RMH/pdf/2004/pdf/Vol72-4-2004-2.pdf

94. García J, Alger J, Padgett D, Rodríguez C, Soto S. Descripción de casos de coinfección dengue y malaria, Hospital Escuela Universitario, Tegucigalpa, Honduras, 2010 - 2014. Rev Med Hondur [Internet]. 2016 [acceso 2 de febrero 2019]; 84(1-2):18-25. Disponible en: http://www.bvs. 
hn/RMH/pdf/2016/pdf/Vol84-1-2-2016-5.pdf

95. Nieto Zelaya VG, Alvarado Claros VG, García JA, Alger J, Tovar Calderón SN, Navarro JJ. Escolar con malaria por Plasmodium falciparum de África: riesgo para la salud pública en Honduras. Rev Med Hond [Internet]. 2019 [acceso 2 de febrero 2019]; 87(1):20-26. Disponible en: http://www.bvs.hn/ $\mathrm{RMH} / \mathrm{pdf} / 2019 / \mathrm{pdf} / \mathrm{Vol} 87-1-2019-6 . \mathrm{pdf}$

96. Lorenzana de Rivera I, Lara C, Sánchez L. Prevalencia de marcadores de hepatitis $\mathrm{B}$ y $\mathrm{C}$ en diferentes grupos poblacionales de la Región Metropolitana de Honduras. Rev Med Hondur [Internet]. 1997 [acceso 2 de febrero 2020]; 65 (1): 12-16. Disponible en: http://www.bvs.hn/RMH/ pdf/1997/pdf/Vol65-1-1997-3.pdf

97. XIII Congreso de la Asociación Centroamericana y del Caribe de Parasitología y Medicina Tropical. Rev Med Hondur 2017; 85 (Suplemento 2): 1-114. http://www.bvs.hn/RMH/pdf/2017/pdf/Vol85-S2-2017.pdf.

98. Martel BI, Corrales Padilla H, Ponce C, de Ponce E. Criociragia como alternativa terapéutica en leishmaniasis cutánea atípica. Rev Med Hondur [Internet]. 1997 [acceso 2 de febrero 2020]; 65(2): 62-64. Disponible en: http://www.bvs.hn/RMH/pdf/1997/pdf/Vol65-2-1997-4.pdf

99. Grinspan S, Garda S, Molina S. Donación Voluntaria de Sangre y Derivados en el I.H.S.S. Un Modelo de Auto-Abastecimiento Institucional. Rev Med Hondur [Internet]. 1991 [acceso 2 de febrero 2020]; 59(3): 118122. Acceso 2 de febrero de 2020. Disponible en: http://www.bvs.hn/RMH/ $\mathrm{pdf} / 1991 / \mathrm{pdf} /$ Vol59-3-1991-3.pdf

100. Anteproyecto para la creación del Banco Nacional de Sangre. Rev Med Hondur [Internet]. 1981 [acceso 2 de febrero 2020]; 49(2):68-71. Disponible en: http://www.bvs.hn/RMH/pdf/1981/pdf/Vol49-2-1981-6.pdf

101. Maldonado Triminio JO. Caracterización de donadores seropositivos por Trypanosoma cruzi y su manejo, Banco de Sangre, Hospital Escuela, Tegucigalpa, 2016 Rev Med Hondur [Internet]. 2019 [acceso 2 de febrero 2020]; 87(1):7-11. Disponible en: http://www.bvs.hn/RMH/pdf/2019/pdf/ Vol87-1-2019-3.pdf

102. Fernández JA, Grinspan S. Detección de la infección por Virus de Inmunodeficiencia Humana en donantes de sangre. Rev Med Hondur [Internet]. 1988 [acceso 2 de febrero 2020]; 56 (2): 143-146. Disponible en: http://www.bvs.hn/RMH/pdf/1988/pdf/Vol56-2-1988-4.pdf

103. Javier Zepeda CA, Villeda Bermúdez R. Mordeduras de serpiente: toxicidad y estudios de laboratorio. Rev Med Hondur [Internet] 1980 [acceso 2 de febrero 2020]; 48(2):39-42. Disponible en: http://www.bvs.hn/ $\mathrm{RMH} / \mathrm{pdf} / 1980 / \mathrm{pdf} /$ Vol48-2-1980-2.pdf

104. Izaguirre GonzálezAI, Matute Martínez CF, Barahona-López DM, SánchezSierra LE, Perdomo Vaquero R. Caracterización clínico-epidemiológica de mordedura de serpiente en el Hospital Regional Santa Teresa de Comayagua, 2014-2015. Rev Med Hondur [Internet]. 2017 [acceso 2 de febrero 2020]; 85(1-2): 21-26 Disponible en: http://www.bvs.hn/RMH/ pdf/2017/pdf/Vol85-1-2-2017-6.pdf

105. Sandoval Cálix W. Caso dramático por mordedura de barba amarilla (Bothrops asper) envenenamiento Grado IV. Rev Med Hondur [Internet]. 1985 [acceso 2 de febrero 2020]; 53(4):304-306. Disponible en: http:// www.bvs.hn/RMH/pdf/1985/pdf/Vol53-4-1985-7.pdf

106. Gutiérrez JM. Tratamiento del Envenenamiento por Mordedura de Serpiente en Centroamérica. Instituto Clodomiro Picado, Facultad de Microbiología, Universidad de Costa Rica, San José, Costa Rica. http:// www.bvs.hn/RMH/pdf/2014/flash/S1/files/res/downloads/page_0040.pdf.

107. Organización Mundial de la Salud. Brote de enfermedad por coronavirus (COVID-19). Organización Mundial de la Salud [Internet]. 2020 [acceso 12 de octubre 2020]; Disponible en https://www.who.int/es/emergencies/ diseases/novel-coronavirus-2019

108. Plataforma Todos Contra el COVID-19. Biblioteca Virtual en Salud de Honduras [Internet]. 2020 [acceso 12 de octubre 2020]; Disponible en http://www.desastres.hn/COVID-19/index4.html

109. Henríquez Rivas O. La Evolución de la Medicina Hondureña a través de la Revista Médica. Rev Med Hondur [Internet]. 2000 [acceso 2 de febrero 2020]; (ed. Esp.):2-4. Disponible en: http://www.bvs.hn/RMH/pdf/2000/pdf/ espcom-2000.pdf

ABSTRACT. This literature review was conducted with the purpose of providing a historical perspective that relates the close linkage of infectology and immunology and its impact on medicine in Honduras, relating to medical practice from its diffuse origins from the colonial era to the present day. Information from Revista Médica Hondureña records and similar national publications was reviewed. Clinical, public health, academic and ethical aspects are presented and discussed in the way of acting professionally where the two specialties converge, highlighting elucubrations, contextual updates, processes, institutions and medical figures that have indelibly marked its development and evolution.

Keywords. Allergy and immunology, Clinical medicine, Honduras, Infectious disease medicine. 